\title{
Actualidad para el diagnóstico del carcinoma ductal in situ: correlación radiopatológica
}

J uan Manuel Calderón N ${ }^{1, a}$, Gustavo Febles ${ }^{2, b}$

\section{RESUMEN}

El carcinoma ductal in situ (CDIS) se encuentra en un grupo heterogéneo de tumores, cuyo diagnóstico se ha visto incrementado con el uso de la mamografía como método de cribado. El sistema de clasificación de Van Nuys, que se basa principalmente en el grado nuclear histológico y la presencia de necrosis, es el sistema más reproducible para la clasificación histopatológica. La anomalía más común que se observa en la mamografía son las microcalcificaciones, coexistiendo con otras lesiones como masas y distorsión arquitectural, que representan lesiones de bajo grado. El diagnóstico inicial debe realizarse mediante anamnesis y examen físico detallado que permita realizar una aproximación a las características morfoestructurales de la lesión, para posteriormente llegar a un acercamiento imagenológico y dinámico mediante resonancia magnética (RM), complementada con técnicas de inmunohistoquímica que caractericen el tumor. La presencia de distribución segmentaria morfológica es típico de malignidad (CDIS). La cinética de las lesiones en el estudio dinámico de la RM varía, siendo patognomónico de CDIS el patrón de washout en la fase de reforzamiento tardío. Sin embargo, el patrón dinámico parece estar correlacionado con los hallazgos mamográficos. Los hallazgos de RM y TC multidetector pueden ser útiles en combinación con la RM de mama para el mapeo preoperatorio. Sin embargo, existen técnicas complementarias como la espectroscopía y la difusión ponderada que mej oran la especificidad de la RM y tienen utilidad en la predicción de respuesta a la quimioterapia adyuvante. Estas aplicaciones futuras podrán mejorar la capacidad de diagnóstico oportuno y opciones de tratamiento.

Palabras clave: Carcinoma ductal in situ; microcalcificaciones; mamografía; resonancia magnética; patología.

\section{Current issues in the diagnosis of ductal carcinoma in situ: a radiopathological correlation}

\begin{abstract}
Ductal carcinoma in situ (DCIS) falls into a heterogeneous group of tumors, whose diagnosis has increased with the use of mammography as screening method. The Van Nuys Prognostic Index, mainly based on histological nuclear grade and presence of necrosis, is the most reproducible histopathological classification system. The most common abnormality observed during a mammography are microcalcifications, which coexist with other lesions such as masses and architectural distortion, and represent low-grade lesions. The initial diagnosis should be performed by anamnesis and a detailed physical examination to help determine the morphostructural characteristics of the lesion. Then an imaging and dynamic approach should be achieved by magnetic resonance imaging (MRI) complemented by immunohistochemistry to characterize the tumor. The presence of morphological segmental distribution is typical of malignancy (DCIS). The kinetics of the lesions using a dynamic MRI varies, with the washout and late enhancement pattern being pathognomonic for DCIS. However, the dynamic pattern seems to be correlated with mammographic findings. Multidetector CT and MRI findings may be useful in combination with breast MRI for preoperative mapping. Nevertheless, there are complementary techniques such as spectroscopy and weighted diffusion that improve the specificity of the MRI and are useful in predicting response to adj uvant chemotherapy. These future applications will improve the ability for early diagnosis and treatment options.
\end{abstract}

Keywords: Ductal carcinoma in situ; microcalcifications; mammography; magnetic resonance imaging; pathology.

1. Especialista en ginecotocología y gestión en servicios de salud, posgrado de especialización en mastología, segundo año.

2. Especialista en imagenología y mastología, profesor de la cátedra de radiología del posgrado de la especialización en mastología.

a. Universidad de Montevideo. Uruguay.

b. Universidad de Montevideo, Presidente de la Sociedad de Radiología. Uruguay. 


\section{INTRODUCCIÓN}

El Carcinoma ductal in Situ (CDIS) no es una única enfermedad, se considera un grupo heterogéneo de lesiones cuya presentación conforma diversas características arquitectónicas, distintas tendencias de crecimiento y múltiples patrones citológicas ${ }^{(1)}$.

Las pacientes con CDIS presentan una proliferación de células epiteliales malignas dentro del sistema ductolobulillar sin evidencia mediante microscopía óptica, de invasión a través de la membrana basal hacia el estroma circundante.

La primera descripción del CDIS fue realizada por Broders en 1932, cuando el examen físico era la única indicación para la biopsia ${ }^{(2)}$. Durante el siglo XX, el CDIS fue relativamente poco frecuente llegando a representar menos del $1 \%$ de todos los canceres mamarios; sin embargo con la aplicación de la mamografía como método de cribado, la incidencia del CDIS ha aumentado con tasas de incidencia ajustada por edad pasando de un 2,4 a 27,7/ 100.000 mujeres entre 1981 y $2001^{(3)}$.

\section{METODOLOGÍA}

Para la búsqueda de información bibliográfica, se utilizó la selección de fuentes primarias (artículos originales) y fuentes secundarias, basándose en revisiones de Ensayos Clínicos con aleatorización, estudios de cohortes, casos y controles realizados por más de un centro. La selección de los diferentes títulos relevantes, se logró mediante búsqueda realizada en PubMed con la aplicación de Medical Subjects Headings (MeSH) y la base de datos Cooperativa del sistema BIREME (LILACS) baj o términos clave: "Ductal Carcinoma In Situ, DCIS, Microcalcifications, Mamography, MRT, Pathology".

Se obtuvieron 21661 ítems relacionados, de los cuales posterior a la aplicación de criterios de inclusión limitado a estudios en seres humanos, criterio cronológico no superior a 10 años de antigüedad y acceso disponible en abstract y free full text, se acotaron a 31 referencias primarias, 6 secundarias y 2 títulos originales de gran impacto.

\section{Características patológicas y clasificación}

Como características del CDIS al no ser una única entidad, la frecuencia de recurrencia local difiere según los estudios de acuerdo con el grado nuclear, la presencia y extensión de la necrosis. Para evaluar el riesgo de recurrencia local, diversos sistemas de clasificación histopatológicos se han desarrollado basándose en criterios conocidos como son el grado nuclear, diferenciación celular, arquitectura de la lesión, presencia de comedonecrosis.

Debido a su heterogenicidad relacionada con el patrón de crecimiento, tipo celular, extensión y comportamiento biológico, en sus inicios se dividió simplificadamente en dos tipos:
- Comedocarcinoma, caracterizado por ser menos diferenciado, con nivel mitótico elevado, presencia de necrosis asociada y mayor expresión del protooncogen c-erb B2 con presencia de aneuploidias lo que le otorga un comportamiento microinvasivo ${ }^{(4)}$.

- No Comedocarcinoma, representado por subtipos: micropapilar, cribiforme y sólido, con menos necrosis y sin tendencia a la calcificación ${ }^{(4,5)}$.

Las calcificaciones en tej ido mamario son precipitaciones de contenido cálcico que junto a otras sales combinadas a proteínas, se hallan inmersas en las secreciones. Las microcalcificaciones finas y granulosas se encuentran asociadas a la forma no comedocarcinoma, mientras que calcificaciones lineales y ramificadas, actúan a modo de molde del espacio intraductal de manera extensa presentándose más en la forma comedocarcinoma ${ }^{(6)}$.

En general las microcalcificaciones del carcinoma in situ se localizan en la lesión, incluso en los focos de invasión, ubicándose en la porción intraductal del carcinoma. Pueden variar de tamaño yendo desde $0,5 \mathrm{~mm}$, visibles en la mamografía, hasta 100 nanometros, vistas por el anatomopatólogo al microscopio. Estas calcificaciones están compuestas fundamentalmente por hidroxiapatita cálcica ${ }^{(7)}$.

Radiológicamente las calcificaciones formadas por restos celulares y material necrótico pueden adoptar diversas formas: moldeadas, lineales, en punto y raya o quebradas (comedocarcinoma- pobremente diferenciado). Las moldeadas, se adaptan al espacio intraductal llegando a tener tamaños de hasta $1 \mathrm{~mm}$ de ancho. En las formas bien diferenciadas, se presentan como calcificaciones redondeadas de tamaño y densidad variable generando dudas diagnósticas a diferenciar de patologías como adenosis esclerosante o la mastopatía fibroquística (CFM) ${ }^{(8)}$.

Si bien no hay consenso acerca de cuanto CDIS de tipo comedo exactamente debe estar presente para clasificar al tumor en este subtipo. Según Lagios, es suficiente cualquier cantidad (10\% 20\%) para considerarlo comedo ${ }^{(9)}$. Sin embargo Silvertein, considera que una lesión es comedo sólo si presenta un $50 \%$ o más de éste componente ${ }^{(10)}$.

Moriya y Silverberg, estipularon que un $70 \%$ de este componente era suficiente ${ }^{(11)}$. Poller hizo alusión de la necrosis de tipo comedo para dividir el CDIS en tres subgrupos. Las lesiones que contenían al menos un $75 \%$ de tipo comedo, se denominaron Comedo Puro, mientras que aquellas con menores proporciones al $5 \%$ se llamaron CDIS No Comedo Puro. Las lesiones sin necrosis de tipo comedo y con menos del $5 \%$ se denominaron CDIS No Comedo. Es así como se pudo correlacionar, marcadores como fracción de fase S y c-erb B2, con las recidivas locales ${ }^{(12)}$.

Para que una lesión se clasifique como CDIS comedo, el patrón de crecimiento debe ser sólido y presentar un alto grado nuclear celular (GN3). Sin embargo cuando hay necrosis comedo, las lesiones con bajo grado nuclear 0 
arquitectura cribiforme o micropapilar pueden designarse necrosis comedo. Según Silverberg clasificó las lesiones CDIS comedo, cuando el $70 \%$ de la lesión era de alto grado con crecimiento sólido y sólo un 8\%de los casos cumplieron estos criterios ${ }^{(13)}$.

Los estudios han investigado la utilidad de la clasificación del CDIS para predecir el riesgo de recurrencia después de terapia conservadora. Sin embargo el subtipo arquitectural no es pronóstico siendo independiente de la presencia de necrosis y del grado histológico. La morfología arquitectónica es un método poco satisfactorio siendo necesario complementar su clasificación con factores que reflejen el potencial biológico de las lesiones individuales ${ }^{(13,14)}$.
El grado nuclear se ha convertido en un indicador biológico fiable, superando a la arquitectura y ha surgido como factor histopatológico para identificar agresividad ${ }^{(13)}$. En un análisis de pacientes con CDIS tratadas quirúrgicamente más radioterapia, el grado nuclear fue el único factor significativo en un análisis multivariable que predijo recidivas locales ${ }^{(14)}$.

Se ha visto que el sistema de clasificación más reproducible, es el de Van Nuys; el cuál se basó en la presencia o ausencia de dos factores predictivos de importancia para recidiva, los cuales son el alto grado nuclear y la necrosis de tipo comedo como reflejo de la patobiología de los tumores.

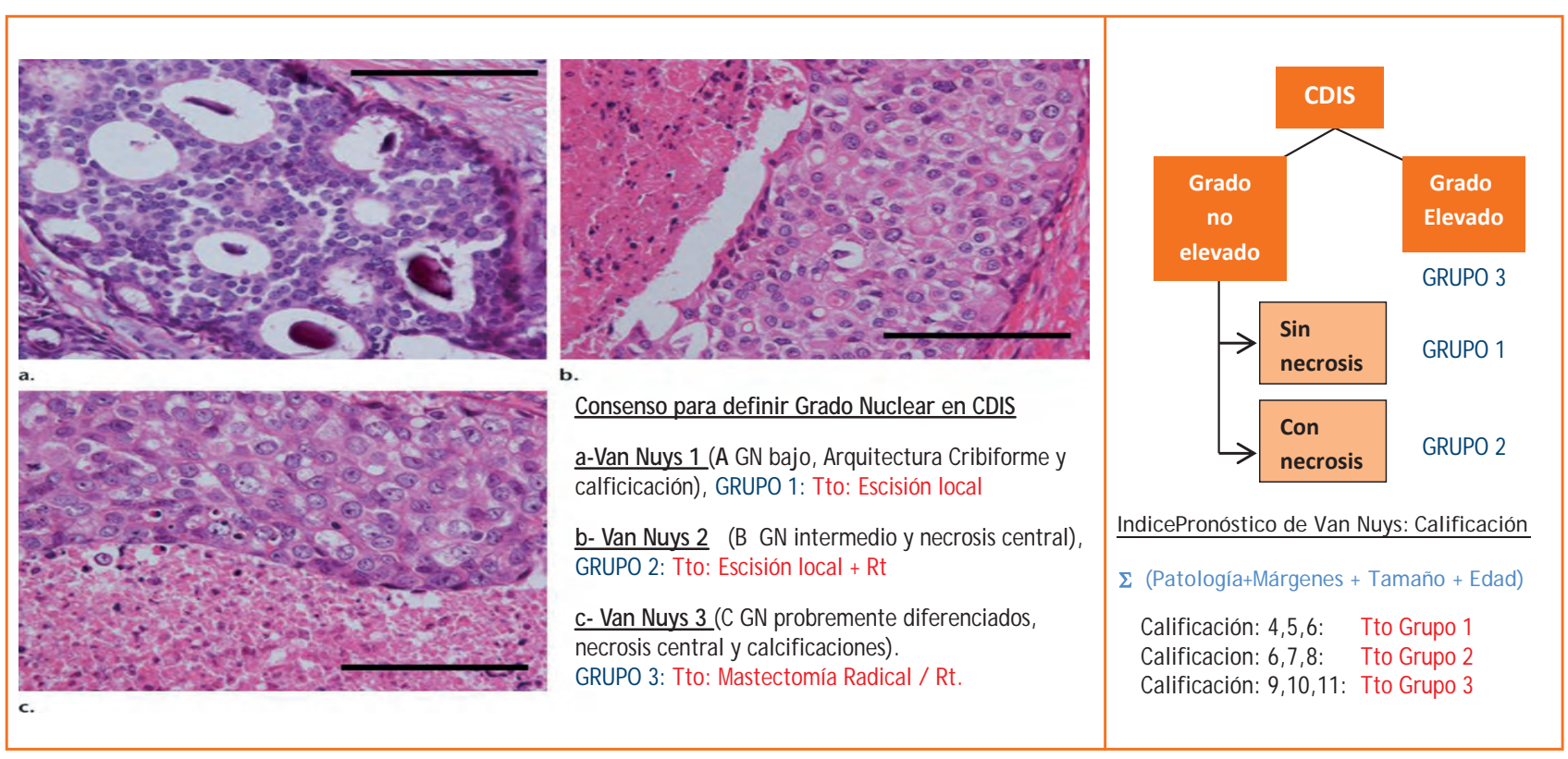

Figura 1. Clasificación y características histopatológicas del carcinoma ductal in situ según Van Nuys ${ }^{(16)}$. Adaptación por Autor: Calderón J M.

Se incluyó para el análisis 4 variables a mencionar:

a) Tamaño tumoral, b) margen tumoral, c) clasificación histopatológica y d) edad de la paciente ${ }^{(15)}$.

El alto grado nuclear como factor más importante en la clasificación Van Nuys, mostró una mayor probabilidad de recidiva después de la conservación de la mama en comparación con las lesiones de bajo grado. De igual forma, las necrosis de tipo comedo se asocian a mal pronóstico ${ }^{(11,16)}$.

\section{Características de la mamografía del CDIS}

Para la detección del CDIS, la mamografía se ha convertido en la principal herramienta a pesar de tener ciertas limitaciones representadas en la sensibilidad de la prueba la cual oscila entre $87 \%$ 95\%

Según Viana (Figura 2), la mamografía como método diagnóstico permite identificar las calcificaciones en sus diversas formas y distribución.

Para el análisis de las microcalcificaciones existen diversos criterios a tener en cuenta: tamaño, número, forma y densidad de distribución. Según el Ministerio de salud Brasilero, las microcalcificaciones de tamaño igual o menor a $0,5 \mathrm{~mm}$ sugieren malignidad, siendo las de mayor tamaño indicativo de benignidad.

Al igual se pudiera inducir en relación al número, ya que a mayor número de microcalcificaciones, mayor sospecha de malignidad $^{(18,19)}$ (Tabla 1). 
Tabla 1. Clasificación morfológica de las calcificaciones ${ }^{(18)}$

\begin{tabular}{ccc}
\multicolumn{3}{c}{ Clasificación morfológica de las calcificaciones } \\
TIPO & MORFOLOGíA & \% Malignidad \\
\hline Tipo I & Anulares, redondeadas, discoides con centro hiperrefringente & Benignas \\
\hline Tipo II & Redondas, isodensas, uniformes & $22 \%$ \\
\hline Tipo III & Puntiformes tipo granulares, difícil identificación & $40 \%$ \\
\hline Tipo IV & Irregulares, poliédricas, en grano de sal & $66 \%$ \\
\hline Tipo V & Vermiculares, ramificadas, con forma de letras & $100 \%$ \\
\hline
\end{tabular}

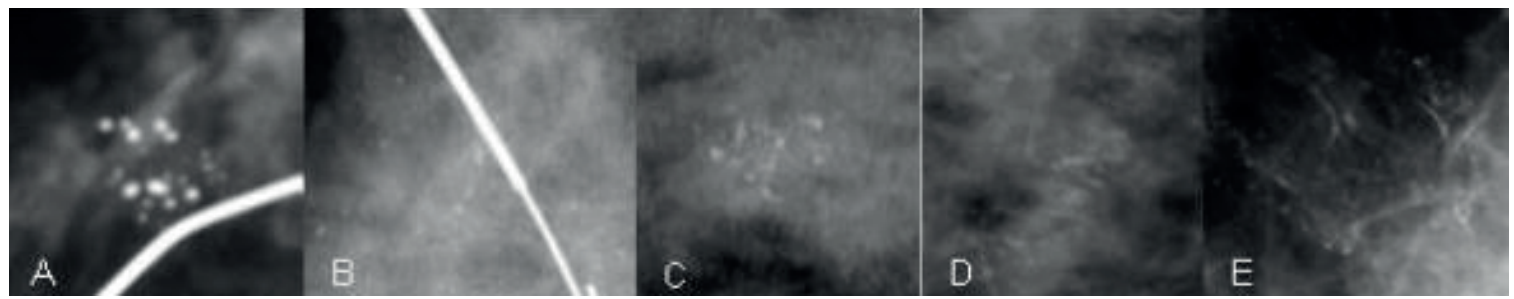

Figura 2. Correlación mamográfica con anatomía patológica (18). Calcificaciones A) Tipo I, B) Tipo II, C) Tipo III, D) Tipo IV, E) Tipo V.

Así mismo la morfología de las microcalcificaciones podrán orientar al clínico ante una probable sospecha de malignidad dentro del encare inicial, que en conjunto a la clínica, la patobiología y el enfoque inmunohistoquímico determinaran el tipo molecular de tumor y orientarán actuación $^{(19)}$.

El nivel de sospecha de CDIS está en función de las características y del número, siendo la mayoría de los carcinomas aquellos que involucran 50 más calcificaciones ${ }^{(20)}$. Sin embargo, Goldstein reportó no encontrar calcificaciones histológicas de hasta un $17 \%$ de los Carcinomas intraductales examinados en hallazgos patológicos de piezas asociadas con recurrencia en mama, después de un tratamiento conservador. Por tanto la ausencia de microcalcificaciones identificables histológicamente, estuvo asociado significativamente con un riesgo aumentado de sufrir recurrencia mamaria ${ }^{(21)}$.

En pacientes con mastectomía es más frecuente la extensión del carcinoma intraductal al pezón o región subareolar con el tipo comedo que con el cribiforme.

Muchos de los carcinomas intraductales detectados por mamografía no constituyen una lesión palpable al examen clínico. Fisher reportó cerca de un $80 \%$ de casos impalpables, incluidos en el Proyecto Nacional de Cirugía de Mama (NSABP). Si bien su tamaño puede estimarse por medio de mamografía con base en la distribución de las calcificaciones, reportándose una subestimación cercana al $23 \%$ de los casos. Además en el estudio, se determinó que las lesiones mayores de $15 \mathrm{~mm}$ eran probablemente multicéntricas ${ }^{(22)}$.

Si bien, las mamografías anormales sin calcificaciones generan sospecha hacia carcinoma de células pequeñas más que de células grandes; las calcificaciones lineales son un marcador de necrosis, mientras que las calcificaciones granulares se pueden asociar con carcinoma intraductal sin necrosis. Estos patrones pueden ser reflejo de condiciones patológicas que conduzcan a distorsiones arquitecturales (cicatrices radiales, esclerosis peri-intersticial al CDIS y la invasión carcinomatosa del Ligamento de Cooper) o masas de tej ido suave que asociados al carcinoma se han reportado en menos del $10 \%$ de los CDIS detectados por mamografía ${ }^{(23)}$.

La mayoría de las lesiones de CDIS se clasifican según la OMS como de bajo grado (grado1), lesiones sin necrosis (Van Nuys 1), siendo más propensos a manifestar anomalías no calcificadas ${ }^{(24)}$.

\section{Otras características imagenológicas}

Puede existir carcinoma intraductal en lesiones radiológicamente circunscritas que parezcan benignas y por tanto ser necesaria la evaluación, mediante técnicas 
complementarias que permitan realzar la captación de contraste (gadolinio) a nivel del parénquima mamario de lesiones detectadas por Resonancia Magnética ${ }^{(25)}$.

Hacia 1971, Mansfield describió por primera vez el comportamiento de muestras de tejido tumoral comparado con tejido normal al medir los tiempos de relajación posterior a la captación de contraste y el seguimiento imagenológico mediante esta técnica ${ }^{(26)}$. En 1983 Yousef publicó resultados a partir de dos pacientes con Ca de mama, usando un imán de 0,03 Teslas (T) lográndose a partir de ese año, el desarrollo y aplicación de bobinas específicas para tejido mamario ${ }^{(26)}$. En 1991 la FDA, aprueba la RM de mama como herramienta de uso complementario a la mamografía, evidenciando una sensibilidad aproximada del $94-100 \%$ para carcinoma invasivo y $50-94 \%$ o $77-96 \%$ según estudios en CDIS, pero con moderada especificidad del $37-97 \%$ de acuerdo a otras ${ }^{(27)}$

Es por tanto que dentro de las variaciones de imágenes propuestas para mejorar la especificidad de la RM, se plantea la aplicación de Espectroscopía, técnica que aporta información sobre la composición bioquímica del tejido estudiado. Su valor se basa en la detección de niveles elevados de Colina, como marcador metabólico tumoral elevado ante la presencia de cáncer de mama ${ }^{(28)}$.

Tiene una sensibilidad del $70-96 \%$ y una especificidad del $67-100 \%$ Adicionalmente permite realizar monitorización de respuesta a quimioterapia en lesiones de $1,5 \mathrm{~cm}$ con una sensibilidad del $89 \%$ y especificidad del $100 \%{ }^{(25,28)}$. Existe como alternativa adicional suplementaria la técnica de difusión ponderada (cuantificación determinada por el valor del coeficiente de difusión aparente), la cual permite la detección de tumores de mama y la distinción entre lesiones benignas y malignas. Se basa en el movimiento aleatorio de las moléculas de agua en los tej idos con alta densidad celular (tej ido tumoral), con una sensibilidad cercana al $90 \%$ y una especificidad del $95,5 \%$ para la detección de lesiones y predicción de respuesta a quimioterapia neo adyuvante ${ }^{(27)}$.

\section{Angiogénesis}

Los tumores malignos presentan un proceso de angiogénesis desorganizado y caótico. Dentro de las características funcionales y morfológicas que permiten identificar diferencialmente entre procesos malignos y benignos, facilitando su detección se encuentran ${ }^{(29)}$ :

- Fragilidad capilar con alta permeabilidad a macromoléculas, tortuosidad vascular y vasodilatación junto a Shunts arterio-venosos, heterogeneidad espacial y estructura caótica con heterogeneidad en la densidad vascular.

En relación a la cinética del reforzamiento tumoral en la RNM, la densidad microvascular de las lesiones malignas juega un papel importante en la determinación de la tasa inicial de absorción media del contraste y la heterogeneidad de realce tumoral. Dentro de las características vasculares incluyen un mayor volumen sanguíneo, formación de Shunts arterio-venosos, incremento de la presión intersticial y mayor permeabilidad capilar ${ }^{(29)}$. Se presentan tres tipos de patrones dinámicos de captación de contraste, con una subdivisión inicial de captación en fase inicial y tardía (Figura 3).

El análisis cinético tiene una sensibilidad de hasta 85-99\% y una especificidad del $40-80 \%$ en la detección de lesiones mamarias.

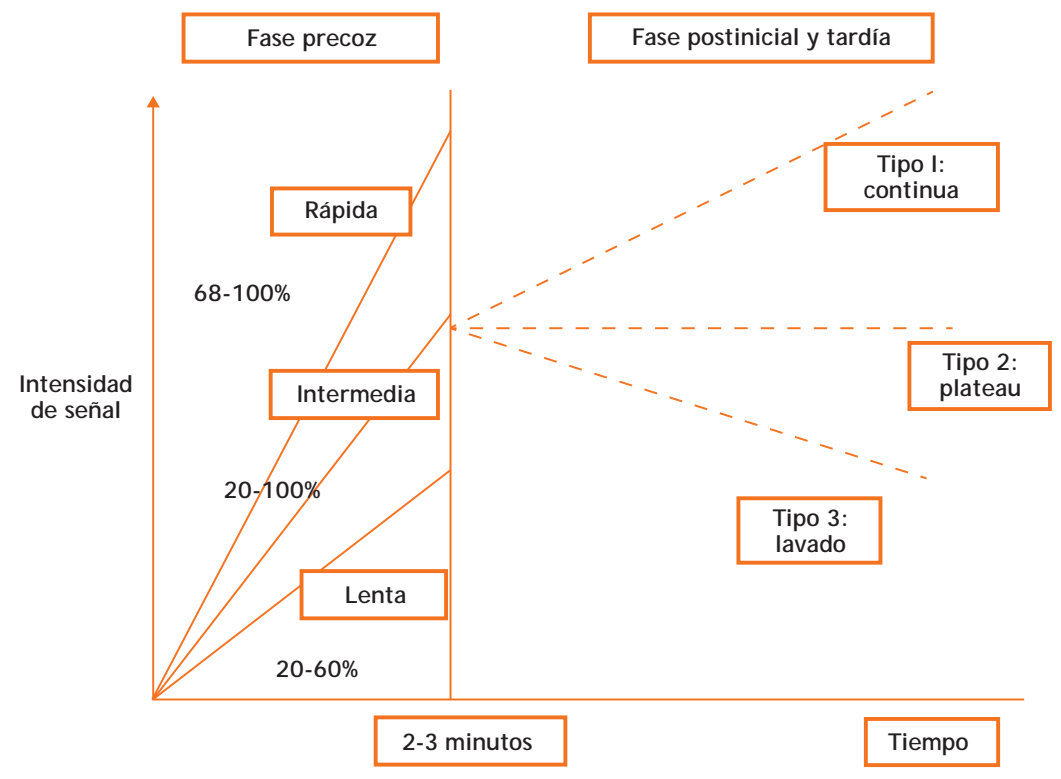

Figura 3. Curvas de tiempo/ intensidad en estudio dinámico de resonancia magnética de mama ${ }^{(29)}$ 
En el patrón de realce Tipo I, benignidad, presenta una sensibilidad del $83 \%$ vs $9 \%$ maligno; en el Tipo II, tiene una sensibilidad del 42,6y especificidad del $75 \%$ para malignidad. El patrón Tipo III, generalmente no se observa en lesiones benignas y tiene una especificidad del $90,4 \%{ }^{(29)}$.

\section{Orientación diagnóstica, seguimiento y tratamiento}

Para el logro del acertado yadecuado diagnóstico, estadificación y tratamiento, es menester contar con una completa anamnesis, así como cronología signológica y sintomática, para posteriormente complementarse con un exhaustivo examen físico que evidencie hallazgos sospechosos (nódulos, secreciones, retracciones, ulceraciones y adenopatías), derivando en la necesidad de solicitar pruebas imagenológicas y toma de muestras adicionales (Punción aspirativa con aguj a fina o biopsia core) para lograr la caracterización inmunogenética. La caracterización de la lesión se debe iniciar mediante el análisis morfológico, para posteriormente complementarlo con el cinético (ACR-Fisher) ${ }^{(30)}$, evidenciándose poscontraste captación intensa y precoz lesional como marcador de malignidad, con relación a señal hipointensa del parénquima (Figura 3). Luego de lograr esta caracterización se busca el patrón inmunohistoquímico correspondiente a cada tumor para así poder plantear una terapéutica y definir el pronóstico y seguimiento individual (Figura 4).

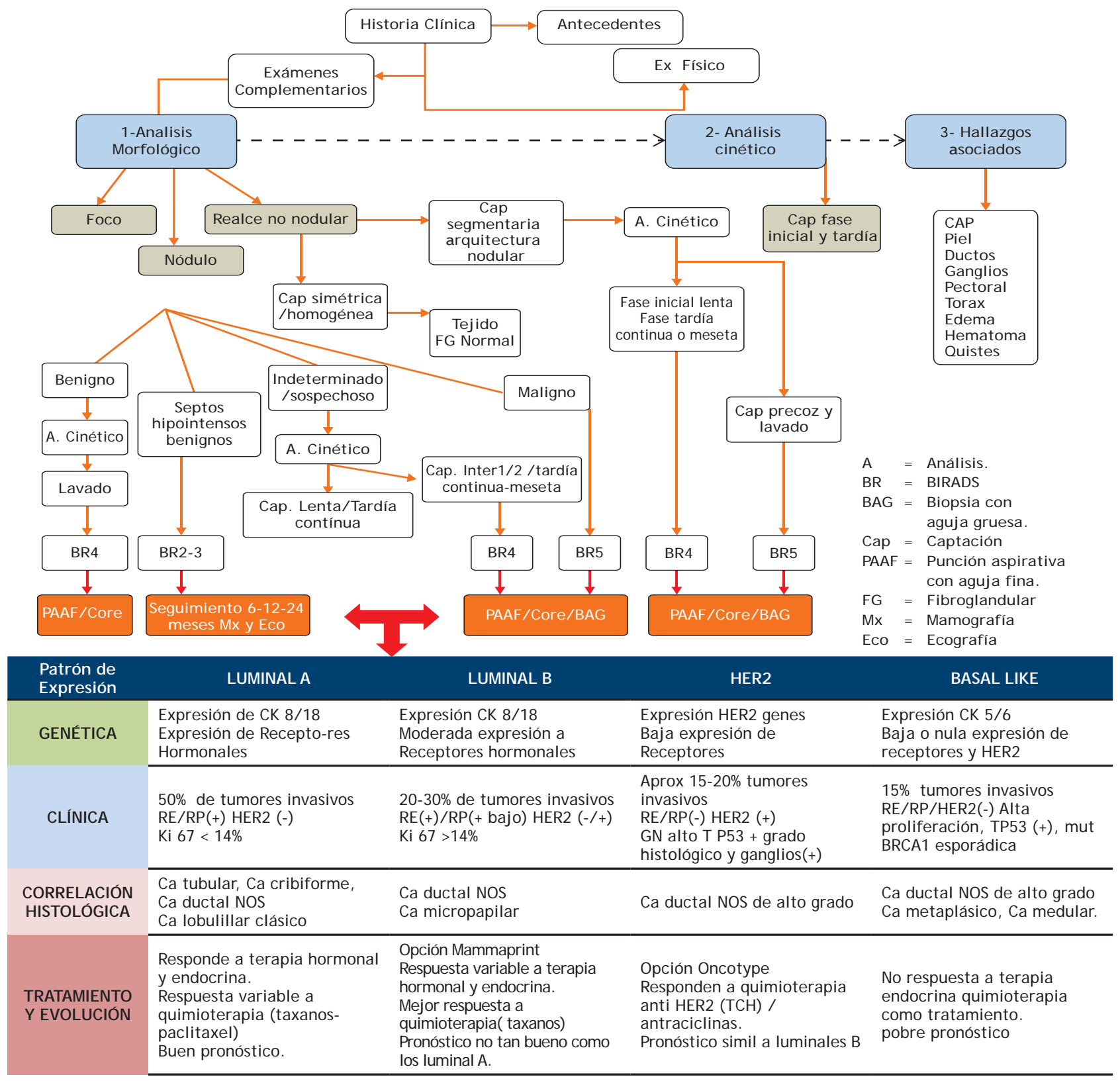

Figura 4. Algoritmo de actuación semiológica, diagnóstica y terapéutica ${ }^{(30,31)}$ Adaptación realizada por autor: Calderón J M. 
A manera de ejemplificar la orientación diagnóstica, se inicia mediante la realización de una entrevista, historia clínica y posterior examen físico, mencionando el caso de una paciente sexo femenino de 44 años, sin historia de patología médica ni quirúrgica relevante, nuligesta por elección, con menarca a los 13 años e inicio de relaciones sexuales 14 años, con ciclos menstruales regulares 28/4 con actual método anticonceptivo de barrera desde hace 10 años y pareja estable de igual data, colpocitología oncológica negativa sin antecedentes de enfermedades infecciosas, nunca mamografía. Consulta por secreción mamaria unilateral derecha de 2 meses de evolución de consistencia espesa, amarronada de escasa cantidad sin fetidez, acompañada de induración en sector supero externo en mama ipsilateral sin antecedentes traumáticos ni otras asociaciones.

Al examen físico a nivel de la mama derecha sector del radio de la hora 12 a $2-3 \mathrm{~cm}$ de la areola, se palpa nódulo indurado, móvil de contornos regulares de $2 \mathrm{~cm}$ de diámetro sin alteraciones cutáneas del complejo areola pezón, evidenciándose a la maniobra de expresión secreción hemática a través de un galactóforo, realizándose extendido citológico y biopsia enviándose a estudio a laboratorio patológico. A nivel axilar no se evidencian hallazgos relevantes, mama contralateral sin alteraciones macroscópicas con resto del examen físico normal.

Vemos como la situación clínica de la paciente nos orienta hacia una mujer en edad reproductiva perimenopáusica, con un cuadro de telorrea unilateral (derrame por pezón) y nódulo mamario palpable.

En la orientación diagnóstica dentro de estudios complementarios solicitados se realizó una mamografía que informó una induración en sector superior hora de radio 12 sin traducción mamográfica por alta radio densidad. Por tal motivo se complementa estudio mediante la realización de una ecografía mamaria que evidencia Imagen sólida mal delimitada, no homogénea de $19 \mathrm{~mm}$ con galactorrea hemática categorizando la lesión como BIRADS 5 (Altamente sugestivo malignidad VVP >95\%) (Figura 5).

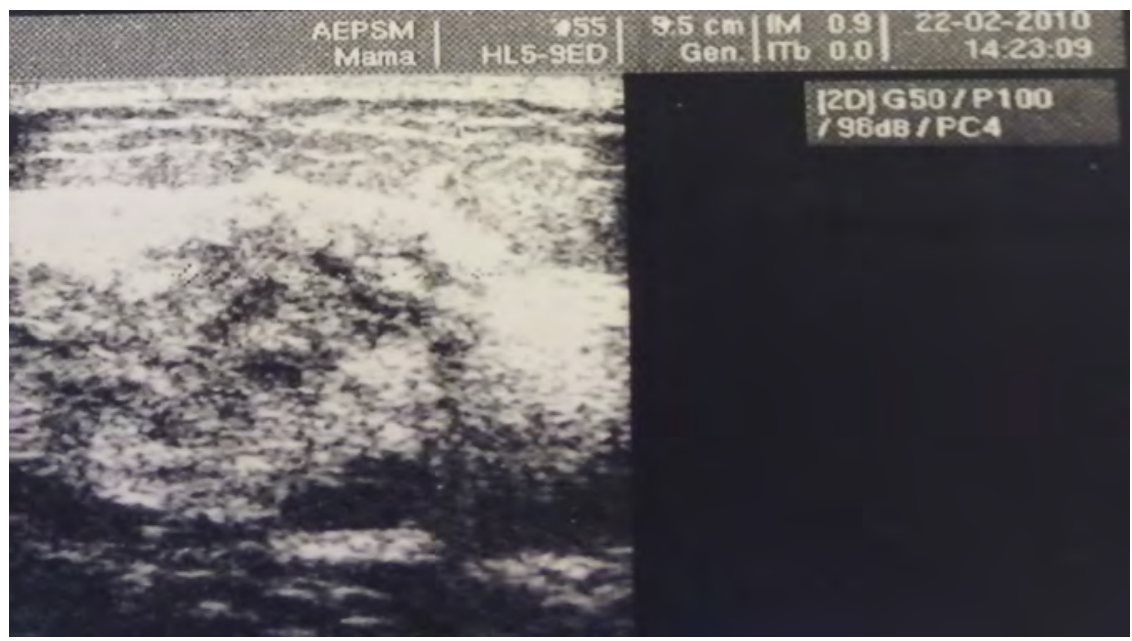

Figura 5. Ecografía mamaria

Es importante aclarar que la densidad mamográfica se ha relacionado con un aumento del riesgo de cáncer de mama siendo aún controvertido, encontrándose inversamente relacionada con la precisión mamográfica y una disminución en su sensibilidad del $10-20 \%^{(32)}$. Por tanto la legislación americana ha incorporado este concepto dentro de los informes mamográficos desde el $2013^{(32)}$.

Radiológicamente la mama contiene 2 componentes que incluyen grasa y tejido fibroglandular. La grasa se caracteriza por tener un bajo coeficiente de atenuación de los rayos $x$ siendo más radiolúcido, mientras que el al to componente fibroglandular asociado a la densidad, podrá evidenciarse como zonas radiopacas interfiriendo con la visualización de detalles imagenológicos ${ }^{(32)}$. La densidad mamaria se define como la proporción existente entre la grasa y el tejido fibroglandular, existiendo una escala de 4 grados de evaluación visual BIRADS (10\% mayoritariamente grasa, $40 \%$ áreas dispersas de densidad fibroglandular, $40 \%$ heterogéneamente densas, y 10\% extremadamente densas) ${ }^{(33,34)}$.

La ecografía se ha convertido en un método diagnóstico complementario a la mamografía para valorar la naturaleza sólida o quística de lesiones en mamas predominantemente densas ${ }^{(35)}$. Tiene una sensibilidad para detectar lesiones papilares de hasta el $83 \%^{(36)}$.

Como se evidenció en la ecografía, pueden encontrarse masas mal definidas e irregulares con bordes indistintos, con densidad hipoecogénica y bordes ecogénicos con sombra acústica posterior como se mostró en el caso (Figura 5). 
Ante la presencia de secreción por pezón constatado durante el examen y realización de ecografía, se procedió a la toma de muestra citológica y biopsia core guiada, reportándose la presencia de un citograma hemorrágico con hematíes alterados, placas de línea epitelial con moderada anisocariosis tipo papilar; llegándose al diagnóstico histopatológico de Carcinoma papilar.

Si bien la citología no se recomienda de manera sistemática en todas las pacientes con telorrea, ya que no es costo efectivo ${ }^{(37)}$, puede presentar una sensibilidad variable 40 $85 \%$ hacia la distinción entre lesiones papilares malignas y benignas ${ }^{(38)}$. La presencia de caracteres citológicos como frotis hipercelulares con grupos papilares y presencia de células aisladas y dispersas con hipercromasia son orientadores al carcinoma ${ }^{(38,39)}$.

Del estudio inmunohistoquímico se informó positivo para receptores estrogénicos y de progesterona, se planteó como estrategia de tratamiento resección sectorial con conservación de piel ${ }^{(16)}$. Se constató con la anatomía patológica definitiva de la pieza quirúrgica la presencia de Carcinoma papilar sólido de 10 mm, GN 2 microinvasor de $1 \mathrm{~mm}$ con Carcinoma Ductal In Situ de bajo grado nuclear. En un Segundo tiempo se realizó vaciamiento axilar ipsilateral (0/3) y concomitantemente se inició hormonoterapia y radioterapia. La paciente se encuentra actualmente en seguimiento por equipo oncológico multidisciplinario.

En conclusión, existe una buena correlación radiopatológica de las microcalcificaciones en los CDIS, siendo predominante para grado I y || la morfología pequeña y redondeada e histología con componente cálcico y grado III, calcificaciones alargadas amorfas de material necrótico intraductal.

El sistema de Van Nuys que incluye el grado nuclear histológico y la presencia de necrosis, es el sistema más reproducible para la clasificación histopatológica.

Dentro de las técnicas complementarias de diagnóstico, la RM se ha convertido en un arma potente complementaria a las técnicas de screening (mamografía) y ecografía. La aplicación de técnicas futuras suplementarias como la espectroscopía y difusión ponderada, podrán en conjunto con la RM, mejorar la capacidad diagnóstica del cáncer de mama más precozmente, incrementando las opciones de tratamiento y el pronóstico de las pacientes.

\section{REFERENCIAS BIBLIOGRÁFICAS}

1. Silverstein MJ. Carcinoma Ductal in Situ. Cáncer de Mama. España: Marban; 2014.

2. Broders AC. Carcinoma in situ contrasted with benign penetrating epithelium. JAMA, 1932; 99:1670-74.

3. Sumner WE, Koniaris LG, Snell SE, Spector S, Powell J , Avisar E, et al. Results of 23,810 cases of ductal carcinoma in situ. Ann Surg Oncol 2007; 14(5):1638-43.

4. Powell DE, Stelleing CB. Carcinoma de Mama. Enfermedades de la Mama. España: Mosby-Doyma libros, S. A, 2007:274-5.
5. Murphy WA, DeSchryver-Kecskemeti K. Isolated clustered microcalcifications in the breast: radiologic-pathologic correlation. Radiology. 1978; 127(2):335-41.

6. Mas J , Balagueró L, De Anta J, et al. Atlas de Patologías: Cancer de Mama. España: Ed Asklepios, 2013: 29-35.

7. Bellahcyene A, Castronovo V. Increased expressions of osteonectin and osteopontin, two bone matrix proteins, in human breast cáncer. Am J Pathol. 1995; 146(1):95-100.

8. Holland R, Hendriks J. Microcalcifications associated with ductal carcinomain situ: mamographic-pathologic correlation. Sem Pathol, 1994; 11(3):181-92.

9. Lagios M, Westdahl PR, Margolin FR, Rose MR. Duct carcinoma in situ: relationship of extent of noninvasive disease to the frecuency of occult invasión,multicentricity, lymph node metastases, and shortterm tretment failures. Cancer.1982; 50(7): 1309.

10. Silverstein MJ, Woo C. Carcinoma Ductal in Situ. La Mama. España: Ed Panamericana, 2007; 48: 1029-63.

11. Moriya T, Silverberg S. Intraductal Carcinoma of the breast. Int J Surg Pathol, 1995; 3:83.

12. Poller D. Ductal Carcinoma in situ breast: a proposal for a new simplified histological classification association between celular proliferation and c-erb-2 protein expression. Mod Pathol, 1995; 7:257.

13. Silverstein MJ . The University of Southern California/Van Nuys prognostic index for ductal carcinoma in situ of the breast. Am J Surg. 2003; 186(4):337-43.

14. Martinez A, Aparicio I, Hernandez G, Muñoz M, de Santiago J . Factores Pronósticos del Carcinoma Ductal in Situ de mama. España. Rev Chil Obstet Ginecol. 2008; 73(1):35-41.

15. Cervantes G, Ochoa F, Ocampo R, Erazo A, Escudero P, Rivera H. Tercera Revisión del Consenso Nacional sobre Diagnóstico y Tratamiento del Cáncer Mamario. Ginecol Obstet, Mex. 2008; 78(2): 138-50.

16. Yamada T. Radiologic-pathologic correlation of ductal carcinoma in situ. Radiographics. 2010;30(5):1183-98.

17. Yang TL, Liang HL, Chou CP, Huang J S, Pan HB. The Adjunctive Digital breast tomosynthesis in diagnosis of breast cáncer. Biomed Research International. Brasil. 2013; 597253:7.

18. Viana AD. Calcificações arredondadas como único achado mamográfico no carcinoma de mama: Correlação mamografiaanatomia patológica. Radiol. Bras.,2005; 38( 3): 163-167.

19. Vianna AD. Cancerização de lóbulos: correlação de achados mamográficos e histológicos. Radiol Bras. 2011; 5(44): 275-78.

20. Aibar L, Santalla A, Lopez-Criado MS, González-Pérez I, Calderón MA, Gallo J L, et al. Clasificación Radiológica y Manejo de las lesiones mamarias. Clin Invest Gin Obst, 2011; 38(4):14149.

21. Goldstein NS, Kestin L, Vicini F. Intraductal carcinoma of the breast. Pathologic features associated with local recurrence in patients treated breast-conserving therapy. Am J Surg Pathol. 2000; 24:1058-67.

22. Coombs J H, Hubbard E. Ductal carcinoma in situ of the breast. Am Surg. 1997; 63:1079-83.

23. Sekine K, Tsunoda-Shimizu H, Kikuchi M, Saida Y, Kawasaki T, Suzuki K. DCIS showing architectural distortion on the screening mammogram: comparison of mammographic and pathological findings. Breast Cancer. 2007; 14(3):281-284.

24. Barreau B, de Mascarel I, Feuga C, MacGrogan G, Dilhuydy MH, Picot $\mathrm{V}$, et al. Mammography of ductal carcinoma in situ of the breast: review of 909 cases with radiographic-pathologic correlations. Eur J Radiol. 2005;54(1):55-61. 
25. Zuñiga I, Villaseñor-Navarro $Y$, Pérez-Badillo M, Cruz-Morales R, Pavón-Hernández C, Aguilar-Cortázar L. Breast Magnetic Resonance and its applications. GAMO, 2012; 4(11):268-80.

26. Kaiser WA. Breast Magnetic Resonance Imaging: Principles and Techiques. Elservier. 2007: 228-235.

27. Sociedad Española de Radiología Médica. Diagnóstico por la Imagen. Cáncer de Mama. Madrid, Radiologia, 2008;2:5-39.

28. Mountford C, Lean C, Malycha P, Russell P. Proton spectroscopy provides accurate pathology on biopsy and in vivo. J Magn Reson Imaging. 2006;24(3):459-477.

29. Herrero J. Resonancia Magnética de mama: estado actual y aplicación clínica. Radiología. 2011;53(1):27-38.

30. Woo C. CDIS: estudios clínicos y controversias en el tratamiento. Cáncer de mama, Ed Marban. 2014; (1): 298-318.

31. Torres S, Acevedo J C, Aguirre B, Aliaga N, Cereceda L, Dagnino B. State of the art in breast cáncer diagnosis and treatment. Revista Médica Clínica Las Condes. 2013; 24(4):588-609 http:/ / dx. doi.org/ 10. 1016/ S0716-8640(13)70199-8.

32. Price E, Hargreaves J, Lipson J, Sickles E, Brenner J, Lindfors $K$. The California Breast Density Information Group: $A$ Collaborative Response to the Issues of Breas
Fuentes de financiamiento:

Este artículo ha sido financiado por los autores.

\section{Conflictos de interés:}

Los autores declaran no tener ningún conflicto de interés.

\section{Correspondencia:}

Nombre: J uan Manuel Calderón Nieto

Dirección: Roque Graseras 743 Apto 902 Código Postal: 11300.

Montevideo, Uruguay.

Teléfono: +598 97061984

Correo electrónico: juanmanuel.calderonnieto@yahoo.es

Recibido: 25 de mayo de 2016

Aprobado: 15 de agosto de 2016 